\title{
Study of Different Levels of Chlorantraniliprole 10\%+Thiamethoxam 20\% Mixture Against Major Insect-pests of Tomato
}

\author{
Sandip Patra ${ }^{1}$, B. C. Das' ${ }^{2}$, S. Sarkar ${ }^{3 *}$, P. P. Dhar ${ }^{2}$ and A. Samanta ${ }^{2}$ \\ ${ }^{1}$ Division of Crop Protection, ICAR Research Complex for NEH Region, Umiam, Meghalaya (793 103), India \\ ${ }^{2}$ Dept. of Agricultural Entomology, BCKV, Mohanpur, Nadia, West Bengal (741 252), India \\ ${ }^{3}$ Krishi Vigyan Kendra, Cooch Behar Uttar Banga Krishi Viswavidyalaya, Pundibari, Coochbehar, W.B. (736 165), India
}

\section{Article History}

Manuscript No. AR1605

Received in $25^{\text {th }}$ May, 2016

Received in revised form $4^{\text {th }}$ October, 2016

Accepted in final form $7^{\text {th }}$ October, 2016

\section{Correspondence to}

*E-mail: surajskrento@gmail.com

\section{Keywords}

Tomato, chlorantraniliprole $10 \%+$ thiamethoxam $20 \%$ mixture, pests

\begin{abstract}
Experiments were conducted at Bidhan Chandra Krishi Viswavidyalaya, Kalyani, West Bengal, India to evaluate the efficacy of chlorantraniliprole $10 \%+$ thiamethoxam 20\%@105, 128 and $150 \mathrm{~g}$ a.i. ha ${ }^{-1}$ along with other insecticides against major pests of tomato under field condition. Tomato crop was raised in Randomized Block Design during 2010-11 and 2011-12 crop seasons. One soil drench application of all the treatments was given at 10 days after transplanting @ $100 \mathrm{ml}^{\text {water plant }}{ }^{-1}$ except $\lambda$-cyhalothrin $5 \mathrm{EC}$ that was given as foliar spray at the same time with three replications. Observations of white fly, leaf miner and fruit borer were taken on $5^{\text {th }}$, $10^{\text {th }}, 15^{\text {th }}, 20^{\text {th }}$ and $30^{\text {th }}$ days after soil drench application. Yield was recorded separately for each treatment during both the seasons. The per cent reduction or increase $(+)$ of parasitoids (Encarsia sp. and Apanteles sp.) were recorded only on $30^{\text {th }}$ day after application. Results revealed that chlorantraniliprole $10 \%+$ thiamethoxam $20 \%$ mixture (a) $150 \mathrm{~g}$ a.i. ha ${ }^{-1}$ gave excellent control of the two sucking pests, viz. leaf miner (5.09 and $5.75 \%)$ and white fly ( 0.50 and 0.41 scale) as well as of Helicoverpa armigera (0.46 and 0.61 larvae plant $\left.^{-1}\right)$ for both the seasons. The yield increases were also significantly higher in Chlorantraniliprole 10\%+thiamethoxam 20\% mixture treatments as well as it showed remarkable specificity to two important natural enemies associated with tomato ecosystem.
\end{abstract}

\section{Introduction}

Chlorantraniliprole $10 \%+$ thiamethoxam $20 \%$ mixture is a broad-spectrum insecticide that employs two complementary modes of action to protect vegetables from both chewing and sucking insects. Its improved activity is due to the combined modes of action, chlorantraniliprole (CTPR), a new mode of action from the anthranilic diamide family of insecticides, and thiamethoxam, a member of the neo-nicotinoid class of chemistry and performs with fewer sprays needed, and helps to deliver improved yields. It has been reported that this mixed formulation having the two modes of action harmonize each other to combat the toughest insects in vegetables that includes a broad range of lepidopteran pests, as well as aphids, whiteflies and flea beetles (Oliveira et al., 2001). Thus, the effects of this need to be tested in combating the notorious insects of various vegetables considering the conservation of the natural enemies. Among the vegetables, tomato is widely known for their outstanding antioxidant content, including rich concentration of Lycopene. It is an important condiment in most diets and a very cheap source of vitamins. It is extensively grown all over the country with an area of 791 thousand ha and production of $15783.2 \mathrm{mt}$ (Anonymous, 2015). Due to tender and supple nature of tomato and its cultivation under high moisture and input regimes, the vegetable is more prone to pest attack. Generally tomato is accounted by a number of insect pests such as tomato fruit borer, blister beetle, aphids, flea beetle, whitefly, leaf miner, red spider mite, thrips etc. Out of which, tomato fruit borer (Helicoverpa armigera Hub.), white fly (Bemisia tabaci Genn.) and leaf miner (Liriomyza trifolii) are most destructive to the crop (Lange and Branson, 1981). Among them fruit borer, Helicoverpa armigera (Hubner) is the most notorious and alone causes yield loss upto 40\% (Tewari and Krishnamoorthy, 1984). Besides direct damage whitefly also causes indirect damage by transmitting Gemini virus to the crop. In India yield loss caused by Gemini virus infestation on tomatoes reached 93.3\% (Sastry and Singh, 1979). Damage by leaf miner on tomato is also increasing day by day in recent years. Worldwide outbreaks of Liriomyza leafminers are 
associated with excessive use of broad-spectrum insecticides (Murphy and La Salle, 1999). To mitigate the losses due to these pests, a huge quantity of pesticides is used in tomato and it is not unusual for the vegetable growers to give $8-10$ sprays in a season without much increase in the yield. Thus, the tomato fruits are likely to retain high level of pesticide residues which may not only be hazardous to consumers but may affect the export quality as well. Further, the excessive reliance on chemicals has led to the problem of development of resistance, resurgence, environmental pollution. Considering all the troubles, the present study was oriented to evaluate chlorantraniliprole $10 \%+$ thiamethoxam $20 \%$ mixture with different doses against major insect pest of tomato.

\section{Materials and Methods}

The experiments were conducted at Bidhan Chandra Krishi Viswavidyalaya, Kalyani, Nadia, West Bengal during 2010-11 and 2011-12 to evaluate the efficacy of mix formulations of chlorantraniliprole $10 \%$ +thiamethoxam $20 \%$ ) against major insect-pests of tomato and its effects on important natural enemies associated with tomato ecosystem. The experiments were conducted in Randomized Block Design (RBD) with three replications. Standard agronomic practices were followed for raising the crop. Tomato seedlings (Variety: Patharkuchi) were transplanted in plot size of $25 \mathrm{~m}^{2}$ with $75 \times 45 \mathrm{~cm}^{2}$ spacing for both the seasons. Treatments viz. chlorantraniliprole 10\%+thiamethoxam 20\% mixture @ 105, 128 and $150 \mathrm{~g}$ a.i. ha $^{-1}$, chlorantraniliprole20 SC @ 50 g a.i. ha ${ }^{-1}$, thiamethoxam 25 WG @100 g a.i. ha ${ }^{-1}$, phorate 10 G @ 1500 g a.i. ha ${ }^{-1}$ and $\lambda$-cyhalothrin $5 \mathrm{EC} @ 15 \mathrm{~g}$ a.i. ha ${ }^{-1}$ were used for experiment. One soil drench application of all the treatments was given at 10 days after transplanting @ $100 \mathrm{ml}$ water plant ${ }^{-1}$ except $\lambda$-cyhalothrin $5 \mathrm{EC}$ that was applied as foliar spray at the same time with knapsack sprayer@ 4001 of water ha ${ }^{-1}$. In case of white fly, number of nymphal stages of white fly/five random leaves from apical portion of five random plants/ plot was counted while per cent leaf damaged by leaf miner was taken into account before as well as on $5^{\text {th }}, 10^{\text {th }}, 15^{\text {th }}, 20^{\text {th }}$ and $30^{\text {th }}$ days after application. For fruit borer, the counts were based on number of larvae present/ten plants before application as well as on $5^{\text {th }}, 10^{\text {th }}, 15^{\text {th }}, 20^{\text {th }}$ and $30^{\text {th }}$ day after application. The number of infested and healthy fruits was counted at each picking. The data on infestation and yield were subjected to analysis of variance after making necessary transformation. Parasitised white fly nymph and Helicoverpa larvae by Encarsia sp. and Apanteles sp. respectively, were recorded from five leaves selected previously at randomly from five random plants on only $30^{\text {th }}$ day after application. The data were subjected to suitable transformation and the critical difference (CD) at $p=0.05$ level of significance was worked out.

\section{Results and Discussion}

\subsection{Effects on incidence of white fly}

The results of white fly incidence are presented in Table 1. During 2010-11, it was observed that all treatments were effectual and superior over control. The mean white fly population was minimum in plots treated with chlorantraniliprole 10\%+thiamethoxam 20\% mixture@ $150 \mathrm{~g}$ a.i. ha ${ }^{-1}$ with lowest scoring (0.5) which was at par with thiamethoxam25 WG @ 100 g a.i. ha ${ }^{-1}(0.56)$ and chlorantraniliprole 10\%+thiamethoxam $20 \%$ mixture (a) $128 \mathrm{~g}$ a.i. ha ${ }^{-1}(0.78)$. Next order of treatments were chlorantraniliprole 10\%+thiamethoxam 20\% mixture @ $105 \mathrm{~g}$ a.i. ha ${ }^{-1}(0.97)$ which was at per with $\lambda$-cyhalothrin 5 EC @ 15 g a.i. ha ${ }^{-1}(1.16)$. This mixture with all doses and thiamethoxam were significantly different from other treatments in reducing white fly population. Chlorantraniliprole20 SC @ 50 g a.i. ha ${ }^{-1}$ and phorate $10 \mathrm{G} @ 1500 \mathrm{~g}$ a.i. ha ${ }^{-1}$ (score 1.19 and 1.50 respectively) were not as effective as chlorantraniliprole $10 \%+$ thiamethoxam $20 \%$ mixture at higher doses and thiamethoxam but superior over control plots (1.78). During 2011-12, similar trend of efficacies were found against this pest and occupied the order of efficacy as chlorantraniliprole 10\%+thiamethoxam 20\% mixture@150 g a.i.ha ${ }^{-1}>$ thiamethoxam25 WG@100 g a.i. ha ${ }^{-1}>$ chlorantraniliprole $10 \%+$ thiamethoxam $20 \%$ mixture (a) 128 g a.i. ha ${ }^{-1}>$ chlorantraniliprole $10 \%$ +thiamethoxam $20 \%$ mixture@105 g a.i. ha ${ }^{-1}>\lambda$-cyhalothrin5 EC @ 15 g a.i. ha ${ }^{-1}$ >chlorantraniliprole20 SC@50 g a.i. ha ${ }^{-1}>$ phorate10 G@ $1500 \mathrm{~g}$ a.i. ha ${ }^{-1}$. Mean of two seasons observation indicated the similar trend of results of individual season and maintained the same trend in the order of efficacy against this pest. Information regarding efficacy of chlorantraniliprole $10 \%+$ thiamethoxam $20 \%$ mixture on white fly is very scanty but many authors reported the effectiveness of thiamethoxam against white fly. Present findings are in conformity with the results of Lee et al. (2002) who reported that thiamethoxam was very effective against adults of white fly when applied at recommended dose. Further the findings were agreed with the study of David and Robert (2002) who reported that thiamethoxam was effective insecticide to control white fly in tomato as well as stated that control appeared to be better with soil application than foliar application.

\subsection{Effects on incidence of leaf miner}

Effects of treatments on leaf miner incidence are depicted in Table 2. Mean per cent leaf damage varied from 5.09 to 12.44 and 5.75 to $13.27 \%$ during $2010-11$ and 2011-12, respectively. All the treatments were significantly superior 
Table 1: Effect of single soil drench application of chlorantraniliprole $10 \%+$ thiamethoxam $20 \%$ mixture on incidence of white fly Bemisia tabaci Genn. on tomato

\begin{tabular}{|c|c|c|c|c|c|c|c|c|c|c|c|c|c|c|}
\hline \multirow[t]{3}{*}{ Treatments } & \multirow{3}{*}{$\begin{array}{l}\text { Dose } \\
(\mathrm{g} \text { a.i. } \\
\left.\mathrm{ha}^{-1}\right)\end{array}$} & \multicolumn{13}{|c|}{ Scoring of white flies on different days after application } \\
\hline & & \multicolumn{6}{|c|}{ First season } & \multicolumn{6}{|c|}{ Second season } & \multirow{2}{*}{$\begin{array}{l}\text { Over- } \\
\text { all } \\
\text { Mean }\end{array}$} \\
\hline & & $\begin{array}{c}5^{\text {th }} \\
\text { DAA }\end{array}$ & $\begin{array}{l}10^{\text {th }} \\
\text { DAA }\end{array}$ & $\begin{array}{l}15^{\text {th }} \\
\text { DAA }\end{array}$ & $\begin{array}{l}20^{\text {th }} \\
\text { DAA }\end{array}$ & $\begin{array}{l}30^{\text {th }} \\
\text { DAA }\end{array}$ & Mean & $\begin{array}{c}5^{\text {th }} \\
\text { DAA }\end{array}$ & $\begin{array}{l}10^{\text {th }} \\
\text { DAA }\end{array}$ & $\begin{array}{l}15^{\text {th }} \\
\text { DAA }\end{array}$ & $\begin{array}{l}20^{\text {th }} \\
\text { DAA }\end{array}$ & $\begin{array}{l}30^{\text {th }} \\
\text { DAA }\end{array}$ & Mean & \\
\hline $\mathrm{C} 10+$ & 105 & $\begin{array}{c}0.73 \\
(0.85)\end{array}$ & $\begin{array}{c}0.69 \\
(0.83)\end{array}$ & $\begin{array}{c}0.84 \\
(0.92)\end{array}$ & $\begin{array}{c}1.14 \\
(1.07)\end{array}$ & $\begin{array}{c}1.44 \\
(1.20)\end{array}$ & $\begin{array}{c}0.97 \\
(0.97)\end{array}$ & $\begin{array}{c}0.69 \\
(0.83)\end{array}$ & $\begin{array}{c}0.63 \\
(0.79)\end{array}$ & $\begin{array}{c}0.72 \\
(0.85)\end{array}$ & $\begin{array}{c}0.93 \\
(0.96)\end{array}$ & $\begin{array}{c}1.20 \\
(1.09)\end{array}$ & $\begin{array}{c}0.83 \\
(0.90)\end{array}$ & 0.90 \\
\hline $\mathrm{C} 10+$ & 128 & $\begin{array}{c}0.56 \\
(0.75)\end{array}$ & $\begin{array}{c}0.45 \\
(0.67)\end{array}$ & $\begin{array}{c}0.54 \\
(0.73)\end{array}$ & $\begin{array}{c}0.69 \\
(0.83)\end{array}$ & $\begin{array}{c}0.84 \\
(0.91)\end{array}$ & $\begin{array}{c}0.62 \\
(0.78)\end{array}$ & $\begin{array}{c}0.48 \\
(0.69)\end{array}$ & $\begin{array}{c}0.39 \\
(0.62)\end{array}$ & $\begin{array}{c}0.57 \\
(0.75)\end{array}$ & $\begin{array}{c}0.63 \\
(0.79)\end{array}$ & $\begin{array}{c}0.72 \\
(0.85)\end{array}$ & $\begin{array}{c}0.56 \\
(0.74)\end{array}$ & 0.59 \\
\hline $\mathrm{C} 10+$ & 150 & $\begin{array}{c}0.46 \\
(0.68)\end{array}$ & $\begin{array}{c}0.36 \\
(0.60)\end{array}$ & $\begin{array}{c}0.45 \\
(0.67)\end{array}$ & $\begin{array}{c}0.57 \\
(0.75)\end{array}$ & $\begin{array}{c}0.66 \\
(0.81)\end{array}$ & $\begin{array}{c}0.50 \\
(0.70)\end{array}$ & $\begin{array}{c}0.36 \\
(0.60)\end{array}$ & $\begin{array}{c}0.27 \\
(0.51)\end{array}$ & $\begin{array}{c}0.42 \\
(0.65)\end{array}$ & $\begin{array}{c}0.47 \\
(0.68)\end{array}$ & $\begin{array}{c}0.54 \\
(0.73)\end{array}$ & $\begin{array}{c}0.41 \\
(0.63)\end{array}$ & 0.45 \\
\hline $\begin{array}{l}\text { Chlorantra- } \\
\text { niliprole } 20 \\
\text { SC }\end{array}$ & 50 & 0.45 & $\begin{array}{c}0.84 \\
(0.92)\end{array}$ & $\begin{array}{c}1.02 \\
(1.01)\end{array}$ & $\begin{array}{c}1.38 \\
(1.17)\end{array}$ & $\begin{array}{l}1.77 \\
(1.33)\end{array}$ & $\begin{array}{l}1.19 \\
(1.08)\end{array}$ & $\begin{array}{c}0.87 \\
(0.93)\end{array}$ & $\begin{array}{c}0.80 \\
(0.89)\end{array}$ & $\begin{array}{c}0.93 \\
(0.96)\end{array}$ & $\begin{array}{c}1.07 \\
(1.03)\end{array}$ & $\begin{array}{c}1.29 \\
(1.14)\end{array}$ & $\begin{array}{c}0.99 \\
(0.99)\end{array}$ & 1.09 \\
\hline $\begin{array}{l}\text { Thiamethox- } \\
\text { am } 25 \text { WG }\end{array}$ & 100 & $\begin{array}{c}0.48 \\
(0.69)\end{array}$ & $\begin{array}{c}0.42 \\
(0.65)\end{array}$ & $\begin{array}{c}0.48 \\
(0.69)\end{array}$ & $\begin{array}{c}0.66 \\
(0.81)\end{array}$ & $\begin{array}{c}0.78 \\
(0.88)\end{array}$ & $\begin{array}{c}0.56 \\
(0.74)\end{array}$ & $\begin{array}{c}0.36 \\
(0.60)\end{array}$ & $\begin{array}{c}0.33 \\
(0.57)\end{array}$ & $\begin{array}{c}0.36 \\
(0.59)\end{array}$ & $\begin{array}{c}0.48 \\
(0.69)\end{array}$ & $\begin{array}{c}0.84 \\
(0.92)\end{array}$ & $\begin{array}{c}0.47 \\
(0.67)\end{array}$ & 0.52 \\
\hline $\begin{array}{l}\text { Phorate } \\
10 \mathrm{G}\end{array}$ & 1500 & $\begin{array}{c}0.84 \\
(0.92)\end{array}$ & $\begin{array}{c}0.84 \\
(0.92)\end{array}$ & $\begin{array}{c}1.44 \\
(1.20)\end{array}$ & $\begin{array}{c}1.98 \\
(1.41)\end{array}$ & $\begin{array}{c}2.40 \\
(1.55)\end{array}$ & $\begin{array}{c}1.50 \\
(1.20)\end{array}$ & $\begin{array}{c}0.75 \\
(0.87)\end{array}$ & $\begin{array}{c}0.80 \\
(0.89)\end{array}$ & $\begin{array}{c}1.17 \\
(1.08)\end{array}$ & $\begin{array}{c}1.44 \\
(1.20)\end{array}$ & $\begin{array}{c}1.84 \\
(1.36)\end{array}$ & $\begin{array}{c}1.20 \\
(1.08)\end{array}$ & 1.35 \\
\hline $\begin{array}{l}\lambda \text { Cyhalo- } \\
\text { thrin } 5 \mathrm{EC}\end{array}$ & 15 & $\begin{array}{c}0.27 \\
(0.52)\end{array}$ & $\begin{array}{c}0.84 \\
(0.91)\end{array}$ & $\begin{array}{c}1.20 \\
(1.09)\end{array}$ & $\begin{array}{c}1.53 \\
(1.24)\end{array}$ & $\begin{array}{c}1.98 \\
(1.41)\end{array}$ & $\begin{array}{c}1.16 \\
(1.03)\end{array}$ & $\begin{array}{c}0.15 \\
(0.38)\end{array}$ & $\begin{array}{c}0.69 \\
(0.83)\end{array}$ & $\begin{array}{c}0.99 \\
(0.99)\end{array}$ & $\begin{array}{c}1.29 \\
(1.13)\end{array}$ & $\begin{array}{c}1.53 \\
(1.24)\end{array}$ & $\begin{array}{c}0.93 \\
(0.91)\end{array}$ & 1.05 \\
\hline Control & - & $\begin{array}{c}1.20 \\
(1.10)\end{array}$ & $\begin{array}{c}1.41 \\
(1.19)\end{array}$ & $\begin{array}{c}1.62 \\
(1.27)\end{array}$ & $\begin{array}{c}1.83 \\
(1.35)\end{array}$ & $\begin{array}{c}2.85 \\
(1.69)\end{array}$ & $\begin{array}{c}1.78 \\
(1.32)\end{array}$ & $\begin{array}{c}1.02 \\
(1.01)\end{array}$ & $\begin{array}{c}1.23 \\
(1.11)\end{array}$ & $\begin{array}{l}1.35 \\
(1.16)\end{array}$ & $\begin{array}{c}1.50 \\
(1.22)\end{array}$ & $\begin{array}{c}2.13 \\
(1.46)\end{array}$ & $\begin{array}{c}1.45 \\
(1.19)\end{array}$ & 1.62 \\
\hline $\mathrm{CD}(p=0.05)$ & - & 0.06 & 0.07 & 0.07 & 0.09 & 0.09 & 0.08 & 0.09 & 0.10 & 0.12 & 0.12 & 0.08 & 0.10 & - \\
\hline
\end{tabular}

C10+T20\%: chlorantraniliprole 10\%+thiamethoxam 20\% mixture Figures in parentheses are square root transformed values; DAA=Days after application

over control as well as varied significantly from each others. During first season, lowest mean leaf damage was recorded in chlorantraniliprole $10 \%+$ thiamethoxam $20 \%$ mixture treated plots (5.09\%) when applied @ $150 \mathrm{~g}$ a.i. ha ${ }^{-1}$ closely followed by thiamethoxam 25 WG $@ 100 \mathrm{~g}$ a.i. ha ${ }^{-1}(5.60 \%)$. This mixture@ $128 \mathrm{~g}$ a.i. ha ${ }^{-1}$ was also effective in reducing leaf damage (6.68\%) followed by phorate $10 \mathrm{G} @ 1500 \mathrm{~g}$ a.i. ha ${ }^{-1}$ (7.46\%), $\lambda$-cyhalothrin $5 \mathrm{EC} \mathrm{(7.56 \% )} \mathrm{and} \mathrm{chlorantraniliprole} 20$ SC (8.11\%) but this mixture @ $105 \mathrm{~g}$ a.i. ha ${ }^{-1}(9.51 \%)$ was found not very effective as higher doses while in control plots $12.44 \%$ leaf damage was recorded. In second season, this product @ $150 \mathrm{~g}$ a.i. ha $\mathrm{f}^{-1}$ again proved it's superiority in suppressing pest population with minimum leaf damage $(5.75 \%)$ followed by thiamethoxam 25 WG@100 g a.i. ha ${ }^{-1}(6.14 \%)$ and chlorantraniliprole 10\%+thiamethoxam 20\% mixture@128 g a.i. ha-1 $(7.42 \%)$. During second season, $\lambda$-cyhalothrin $5 \mathrm{EC}$ $(8.59 \%)$ exhibited better result as compared to phorate $10 \mathrm{G}$ (8.64\%). Others treatments showed similar trends of efficacies as recorded in first season. Overall, mean leaf damage was also the lowest in chlorantraniliprole $10 \%$ +thiamethoxam $20 \%$ mixture treated plots (5.42\%) when applied @ 150 g a.i. ha ${ }^{-1}$ followed by thiamethoxam 25 WG (5.87\%), same mixture @ $128 \mathrm{~g}$ a.i. ha ${ }^{-1}(7.05 \%)$, phorate $10 \mathrm{G}(8.05 \%), \lambda$-cyhalothrin 5 EC $(8.10 \%)$, chlorantraniliprole $20 \mathrm{SC}(8.40 \%)$ and same mixture@105 g a.i. ha ${ }^{-1}$ while in control plots leaf damage was $12.85 \%$. Soil application of thiamethoxam resulted in significant reduction of L. trifolii (David and Robert, 2002) that corroborate these findings. Thiamethoxam confers the effectiveness of chlorantraniliprole 10\%+thiamethoxam $20 \%$ mixture to suppress the leaf miner. Findings are in similar with Vyas and Saxena (1981) who reported that phorate @ $0.5 \mathrm{~kg}$ a.i. ha ${ }^{-1}$ or seed treatment $(4 \%)$ with phorate was effective in reducing the damage by leaf miner, Phytomyza horticola up to 14 weeks age of the crop in pea.

\subsection{Effects on incidence of fruit borer, helicoverpa armigera $h u b$}

Table 3 represents the effect of different treatments on incidence of tomato fruit borer (Helicoverpa armigera Hub.). Results revealed that mean population ranged from 0.46 to 3.42 and 0.58 to 4.04 larvae plant ${ }^{-1}$ during $2010-11$ and 2011-12, respectively. Both the seasons all treatments were significantly superior over untreated control and different 
Table 2: Effect of single soil drench application of Chlorantraniliprole $10 \%+$ Thiamethoxam $20 \%$ mixture on incidence of leaf miner Liriomyza trifolii Burgess

\begin{tabular}{|c|c|c|c|c|c|c|c|c|c|c|c|c|c|c|}
\hline \multirow[t]{3}{*}{$\mathrm{T}$} & \multirow{3}{*}{$\begin{array}{l}\text { Dose } \\
(\mathrm{g} \text { a.i. } \\
\left.\mathrm{ha}^{-1}\right)\end{array}$} & \multicolumn{13}{|c|}{ Per cent leaf damage on different days after application } \\
\hline & & \multicolumn{6}{|c|}{ First season } & \multicolumn{6}{|c|}{ Second season } & \multirow{2}{*}{$\begin{array}{c}\text { Over- } \\
\text { all } \\
\text { Mean }\end{array}$} \\
\hline & & $\begin{array}{c}5^{\text {th }} \\
\text { DAA }\end{array}$ & $\begin{array}{c}10^{\text {th }} \\
\text { DAA }\end{array}$ & $\begin{array}{c}15^{\text {th }} \\
\text { DAA }\end{array}$ & $\begin{array}{c}20^{\text {th }} \\
\text { DAA }\end{array}$ & $\begin{array}{c}30^{\text {th }} \\
\text { DAA }\end{array}$ & $n$ & $\begin{array}{c}5^{\text {th }} \\
\text { DAA }\end{array}$ & $\begin{array}{c}10^{\text {th }} \\
\text { DAA }\end{array}$ & $\begin{array}{c}15^{\text {th }} \\
\text { DAA }\end{array}$ & $\begin{array}{c}20^{\text {th }} \\
\text { DAA }\end{array}$ & $\begin{array}{c}30^{\text {th }} \\
\text { DAA }\end{array}$ & n & \\
\hline 1 & 105 & $\begin{array}{c}6.99 \\
(15.33)\end{array}$ & $\begin{array}{c}7.29 \\
(15.66)\end{array}$ & $\begin{array}{c}8.49 \\
(16.94)\end{array}$ & $\begin{array}{c}10.89 \\
(19.27)\end{array}$ & $\begin{array}{c}13.89 \\
(21.88)\end{array}$ & $\begin{array}{c}9.51 \\
(17.82)\end{array}$ & $\begin{array}{c}7.50 \\
(15.89)\end{array}$ & $\begin{array}{c}7.92 \\
(16.35)\end{array}$ & $\begin{array}{c}9.33 \\
(17.79)\end{array}$ & $\begin{array}{c}10.02 \\
(18.45)\end{array}$ & $\begin{array}{c}12.00 \\
(20.27)\end{array}$ & $\begin{array}{c}9.35 \\
(17.75)\end{array}$ & \\
\hline 2 & 128 & $\begin{array}{c}5.40 \\
(13.44)\end{array}$ & $\begin{array}{c}5.46 \\
(13.51)\end{array}$ & $\begin{array}{c}6.06 \\
(14.25)\end{array}$ & $\begin{array}{c}7.68 \\
(16.09)\end{array}$ & $\begin{array}{c}8.79 \\
(17.25)\end{array}$ & $\begin{array}{c}6.68 \\
(14.91)\end{array}$ & $\begin{array}{c}5.49 \\
(13.55)\end{array}$ & $\begin{array}{c}5.70 \\
(13.81)\end{array}$ & $\begin{array}{c}7.80 \\
(16.22)\end{array}$ & $\begin{array}{c}8.79 \\
(17.25)\end{array}$ & $\begin{array}{c}9.33 \\
(17.79)\end{array}$ & $\begin{array}{c}7.42 \\
(15.72)\end{array}$ & 7.05 \\
\hline 3 & 150 & $\begin{array}{c}4.02 \\
(11.57)\end{array}$ & $\begin{array}{c}3.99 \\
(11.52)\end{array}$ & $\begin{array}{c}4.50 \\
(12.25)\end{array}$ & $\begin{array}{c}5.94 \\
(14.11)\end{array}$ & $\begin{array}{c}6.99 \\
(15.33)\end{array}$ & $\begin{array}{c}5.09 \\
(12.96)\end{array}$ & $\begin{array}{c}4.59 \\
(12.37)\end{array}$ & $\begin{array}{c}4.29 \\
(11.95)\end{array}$ & $\begin{array}{c}5.40 \\
(13.44)\end{array}$ & $\begin{array}{c}6.66 \\
(14.96)\end{array}$ & $\begin{array}{c}7.80 \\
(16.22)\end{array}$ & $\begin{array}{r}5 . \\
(13\end{array}$ & 5.42 \\
\hline 4 & 50 & $\begin{array}{r}5.94 \\
(14.1\end{array}$ & $\begin{array}{c}6.06 \\
(14.25)\end{array}$ & $\begin{array}{c}7.80 \\
(16.22)\end{array}$ & $\begin{array}{c}8.79 \\
(17.25)\end{array}$ & $\begin{array}{c}11.94 \\
(20.21)\end{array}$ & $\begin{array}{c}8.11 \\
(16.41)\end{array}$ & $\begin{array}{c}6.33 \\
(14.57)\end{array}$ & $\begin{array}{c}6.99 \\
(15.33)\end{array}$ & $\begin{array}{r}8 . \\
(17 .\end{array}$ & $\begin{array}{c}9.06 \\
(17.52)\end{array}$ & $\begin{array}{c}12.33 \\
(20.56)\end{array}$ & $\begin{array}{c}8.70 \\
(17.05)\end{array}$ & 8.40 \\
\hline 5 & 100 & $\begin{array}{c}4.89 \\
(12.78)\end{array}$ & $\begin{array}{c}4.23 \\
(11.87)\end{array}$ & $\begin{array}{c}4.77 \\
(12.62)\end{array}$ & $\begin{array}{c}5.70 \\
(13.81)\end{array}$ & $\begin{array}{c}8.40 \\
(16.85)\end{array}$ & $\begin{array}{c}5.60 \\
(13.59)\end{array}$ & $\begin{array}{c}5.53 \\
(13.60)\end{array}$ & $\begin{array}{c}4.53 \\
(12.29)\end{array}$ & $\begin{array}{r}5 . \\
(13 .\end{array}$ & $\begin{array}{c}6.39 \\
(14.64)\end{array}$ & $\begin{array}{c}9.03 \\
(17.49)\end{array}$ & $\begin{array}{r}6 . \\
(14\end{array}$ & 5.87 \\
\hline 6 & 1500 & $\begin{array}{c}5.49 \\
(13.55)\end{array}$ & $\begin{array}{c}5.22 \\
(13.21)\end{array}$ & $\begin{array}{c}6.03 \\
(14.21)\end{array}$ & $\begin{array}{c}8.49 \\
(16.94)\end{array}$ & $\begin{array}{c}12.09 \\
(20.35)\end{array}$ & $\begin{array}{c}7.46 \\
(15.65)\end{array}$ & $\begin{array}{c}5.94 \\
(14.11)\end{array}$ & $\begin{array}{c}5.79 \\
(13.92)\end{array}$ & $\begin{array}{c}7.59 \\
(15.99)\end{array}$ & $\begin{array}{c}9.99 \\
(18.43)\end{array}$ & $\begin{array}{c}13.89 \\
(21.88)\end{array}$ & $\begin{array}{c}8.64 \\
(16.87)\end{array}$ & 8.05 \\
\hline 7 & 15 & $\begin{array}{c}2.19 \\
(8.51)\end{array}$ & $\begin{array}{c}4.20 \\
(11.83)\end{array}$ & $\begin{array}{c}6.63 \\
(14.92)\end{array}$ & $\begin{array}{c}9.99 \\
(18.43)\end{array}$ & $\begin{array}{c}14.79 \\
(22.62)\end{array}$ & $\begin{array}{c}7.56 \\
(15.26)\end{array}$ & $\begin{array}{c}2.70 \\
(9.46)\end{array}$ & $\begin{array}{c}5.94 \\
(14.11)\end{array}$ & $\begin{array}{c}7.92 \\
(16.35)\end{array}$ & $\begin{array}{c}11.40 \\
(19.73)\end{array}$ & $\begin{array}{c}15.00 \\
(22.79)\end{array}$ & $\begin{array}{c}8.59 \\
(16.49)\end{array}$ & 8.10 \\
\hline 8 & - & $\begin{array}{c}9.93 \\
(18.37)\end{array}$ & $\begin{array}{c}10.80 \\
(19.19)\end{array}$ & $\begin{array}{c}12.66 \\
(20.84)\end{array}$ & $\begin{array}{c}13.80 \\
(21.18)\end{array}$ & $\begin{array}{c}15.00 \\
(22.79)\end{array}$ & $\begin{array}{c}12.44 \\
(20.47)\end{array}$ & $\begin{array}{c}11.04 \\
(19.41)\end{array}$ & $\begin{array}{c}11.70 \\
(20.00)\end{array}$ & $\begin{array}{c}12.99 \\
(21.13)\end{array}$ & $\begin{array}{c}14.43 \\
(22.33)\end{array}$ & $\begin{array}{c}16.20 \\
(23.73)\end{array}$ & $\begin{array}{c}13.27 \\
(21.32)\end{array}$ & 12.85 \\
\hline${ }^{*} \mathrm{CD}$ & - & 0.17 & 0.16 & 0.26 & 0.22 & 0.13 & 0.18 & 0.18 & 0.27 & 0.20 & 0.15 & 0.16 & 0.19 & - \\
\hline
\end{tabular}

T: Treatments; 1: C10+T20\%; 2: C10+T20\%; 3: C10+T20\%; 4: Chlorantraniliprole 20 SC; 5: Thiamethoxam 25 WG; 6: Phorate $10 \mathrm{G} ; 7$ : $\lambda$-cyhalothrin $5 \mathrm{EC}$; 8 : Control; ${ }^{*} \mathrm{CD}$ : $\mathrm{CD}(p=0.05)$; Figures in parentheses are angular transformed values; $\mathrm{DAA}=$ Days after application

among the individuals. In first season, chlorantraniliprole 10\%+thiamethoxam 20\% mixture @ 150 g a.i. ha ${ }^{-1}$ recorded least number of Helicoverpa larvae (0.46 larvae plant $\left.{ }^{-1}\right)$ followed by chlorantraniliprole $20 \mathrm{SC}\left(0.64\right.$ larvae plant $\left.{ }^{-1}\right)$. Next order of treatments were same mixture @ $128 \mathrm{~g}$ a.i. ha $^{-1}$ and @ 105 g a.i. ha ${ }^{-1}$, phorate $10 \mathrm{G}$, thiamethoxam 20 WG, $\lambda$-cyhalothrin 5 EC with 1.11, 1.85, 1.97, 2.78, and 3.03 larvae plant ${ }^{-1}$, respectively whereas in control plot population was 3.42 larvae plant ${ }^{-1}$. During second season, the best result was recorded with chlorantraniliprole $20 \mathrm{SC}$ ( 0.58 larvae plant $^{-1}$ ) which was closely at par with chlorantraniliprole 10\%+thiamethoxam 20\% mixture @ 150 g a.i. ha ${ }^{-1}(0.61$ larvae plant $\left.{ }^{-1}\right)$. Rest of the treatments maintained their effectiveness accordingly as recorded in first season. Overall, mean larval population of Helicoverpa armigera Hub. was found to be minimum (0.54 larvae plant $\left.{ }^{-1}\right)$ in plots treated with higher dose of chlorantraniliprole $10 \%$ +thiamethoxam $20 \%$ mixture followed by chlorantraniliprole $20 \mathrm{SC}(0.61$ larvae plant ${ }^{-1}$ ) and same mixture @ 128 and 105 g a.i. ha ${ }^{-1}$ with 1.09 and 1.72 larvae plant ${ }^{-1}$, respectively. Higher mean larval populations were found in phorate $10 \mathrm{G}, \lambda$-cyhalothrin
5 EC and thiamethoxam 25 WG with $2.12,3.17$ and 3.24 larvae plant ${ }^{-1}$, respectively while in control plot it was very high (3.73 larvae plant $\left.{ }^{-1}\right)$. Results are in concurrence with the findings of Chowdhary et al. (2010) who reported that rynaxypyr (chlorantraniliprole) was superior in recording less larval populations of Helicoverpa armigera Hub., lower fruit damage and higher fruit yield in okra. Effectiveness of rynaxypyr against lepidopteran pests was already reported earlier by Boseli and Ceredi (2010). Chlorantraniliprole has also proved its superiority against Helicoverpa armigera Hub. in tomato. Many reports have been published regarding efficacy of chlorantraniliprole against different lepidopteran pests which are in conformity with the present findings (Hannig et al., 2009; Yang et al., 2010).

\subsection{Yield}

Yield of tomato during first and second seasons are depicted in Table 3. Maximum yield was recorded in plots treated with chlorantraniliprole 10\%+thiamethoxam 20\% mixture @ 150 g a.i. ha ${ }^{-1}$ (215.06 and 214.06 q ha $^{-1}$ ) followed by same chemical@128 g a.i. ha ${ }^{-1}$ (214.06 and 213.66 q ha $\left.^{-1}\right)$ and chlorantraniliprole20 SC @ 50 g a.i. ha ${ }^{-1}$ (213.66 and 212.06 


\begin{tabular}{|c|c|c|c|c|c|c|c|c|c|c|c|c|c|}
\hline \multirow[t]{3}{*}{$\mathrm{T}$} & \multirow{3}{*}{$\begin{array}{c}\text { Dose } \\
(\mathrm{g} \text { a.i. } \\
\left.\text { ha }^{-1}\right)\end{array}$} & \multicolumn{9}{|c|}{ Number of Helicoverpa armigera larvae plant ${ }^{-1}$} & \multicolumn{3}{|c|}{ Yield $\left(\mathrm{q} \mathrm{ha} \mathrm{h}^{-1}\right)$} \\
\hline & & \multicolumn{4}{|c|}{ First season* } & \multicolumn{4}{|c|}{ Second season $^{*}$} & \multirow{2}{*}{$\begin{array}{c}\text { Overall } \\
\text { mean }\end{array}$} & \multirow{2}{*}{$\begin{array}{c}\text { First } \\
\text { season }\end{array}$} & \multirow{2}{*}{$\begin{array}{l}\text { Second } \\
\text { season }\end{array}$} & \multirow[t]{2}{*}{ Mean } \\
\hline & & $\begin{array}{c}15^{\text {th }} \\
\text { DAA }\end{array}$ & $\begin{array}{c}20^{\text {th }} \\
\text { DAA }\end{array}$ & $\begin{array}{c}30^{\text {th }} \\
\text { DAAA }\end{array}$ & $\overline{\text { Mean }}$ & $\begin{array}{c}15^{\text {th }} \\
\text { DAA }\end{array}$ & $\begin{array}{c}20^{\text {th }} \\
\text { DAA }\end{array}$ & $\begin{array}{c}30^{\text {th }} \\
\text { DAA }\end{array}$ & Mean & & & & \\
\hline 1 & 105 & $\begin{array}{c}0.96 \\
(0.98)\end{array}$ & $\begin{array}{c}1.80 \\
(1.34)\end{array}$ & $\begin{array}{c}2.79 \\
(1.67)\end{array}$ & $\begin{array}{c}1.85 \\
(1.33)\end{array}$ & $\begin{array}{c}1.08 \\
(1.04)\end{array}$ & $\begin{array}{c}1.56 \\
(1.25)\end{array}$ & $\begin{array}{c}2.13 \\
(1.46)\end{array}$ & $\begin{array}{c}1.59 \\
(1.25)\end{array}$ & 1.72 & 212.33 & 212.06 & 212.19 \\
\hline 2 & 128 & $\begin{array}{c}0.69 \\
(0.83)\end{array}$ & $\begin{array}{c}0.99 \\
(0.99)\end{array}$ & $\begin{array}{c}1.65 \\
(1.28)\end{array}$ & $\begin{array}{c}1.11 \\
(1.03)\end{array}$ & $\begin{array}{c}0.66 \\
(0.81)\end{array}$ & $\begin{array}{c}0.99 \\
(0.99)\end{array}$ & $\begin{array}{c}1.59 \\
(1.26)\end{array}$ & $\begin{array}{c}1.08 \\
(1.02)\end{array}$ & 1.09 & 214.06 & 213.66 & 213.86 \\
\hline 3 & 150 & $\begin{array}{c}0.06 \\
(0.24)\end{array}$ & $\begin{array}{c}0.26 \\
(0.51)\end{array}$ & $\begin{array}{c}1.05 \\
(1.02)\end{array}$ & $\begin{array}{c}0.46 \\
(0.59)\end{array}$ & $\begin{array}{c}0.36 \\
(0.60)\end{array}$ & $\begin{array}{c}0.48 \\
(0.69)\end{array}$ & $\begin{array}{c}0.99 \\
(0.99)\end{array}$ & $\begin{array}{c}0.61 \\
(0.76)\end{array}$ & 0.54 & 215.06 & 214.06 & 214.56 \\
\hline 4 & 50 & $\begin{array}{c}0.26 \\
(0.51)\end{array}$ & $\begin{array}{c}0.66 \\
(0.81)\end{array}$ & $\begin{array}{c}0.99 \\
(0.99)\end{array}$ & $\begin{array}{c}0.64 \\
(0.77)\end{array}$ & $\begin{array}{c}0.30 \\
(0.55)\end{array}$ & $\begin{array}{c}0.60 \\
(0.77)\end{array}$ & $\begin{array}{c}0.84 \\
(0.92)\end{array}$ & $\begin{array}{c}0.58 \\
(0.75)\end{array}$ & 0.61 & 213.66 & 212.06 & 212.86 \\
\hline 5 & 100 & $\begin{array}{c}1.95 \\
(1.40)\end{array}$ & $\begin{array}{c}2.73 \\
(1.65)\end{array}$ & $\begin{array}{c}3.66 \\
(1.91)\end{array}$ & $\begin{array}{c}2.78 \\
(1.65)\end{array}$ & $\begin{array}{c}2.79 \\
(1.67)\end{array}$ & $\begin{array}{c}3.96 \\
(1.99)\end{array}$ & $\begin{array}{c}4.35 \\
(2.09)\end{array}$ & $\begin{array}{c}3.70 \\
(1.92)\end{array}$ & 3.24 & 211.66 & 209.66 & 210.66 \\
\hline 6 & 1500 & $\begin{array}{c}1.33 \\
(1.15)\end{array}$ & $\begin{array}{c}1.89 \\
(1.370\end{array}$ & $\begin{array}{c}2.70 \\
(1.64)\end{array}$ & $\begin{array}{c}1.97 \\
(0.93)\end{array}$ & $\begin{array}{c}1.02 \\
(1.01)\end{array}$ & $\begin{array}{c}2.19 \\
(1.48)\end{array}$ & $\begin{array}{c}3.60 \\
(1.90)\end{array}$ & $\begin{array}{c}2.27 \\
(1.46)\end{array}$ & 2.12 & 209.33 & 208.33 & 208.83 \\
\hline 7 & 15 & $\begin{array}{c}2.03 \\
(1.42)\end{array}$ & $\begin{array}{c}3.06 \\
(1.75)\end{array}$ & $\begin{array}{c}3.99 \\
(2.00)\end{array}$ & $\begin{array}{c}3.03 \\
(1.72)\end{array}$ & $\begin{array}{c}2.97 \\
(1.72)\end{array}$ & $\begin{array}{c}3.03 \\
(1.74)\end{array}$ & $\begin{array}{c}3.93 \\
(1.98)\end{array}$ & $\begin{array}{c}3.31 \\
(1.81)\end{array}$ & 3.17 & 208.66 & 207.06 & 207.86 \\
\hline 8 & - & $\begin{array}{c}2.33 \\
(1.53)\end{array}$ & $\begin{array}{c}3.66 \\
(1.91)\end{array}$ & $\begin{array}{c}4.26 \\
(2.06)\end{array}$ & $\begin{array}{c}3.42 \\
(1.83)\end{array}$ & $\begin{array}{c}3.33 \\
(1.82)\end{array}$ & $\begin{array}{c}3.99 \\
(2.00)\end{array}$ & $\begin{array}{c}4.80 \\
(2.19)\end{array}$ & $\begin{array}{c}4.04 \\
(2.00)\end{array}$ & 3.73 & 199.33 & 196.66 & 197.99 \\
\hline${ }^{*} \mathrm{CD}$ & - & 0.05 & 0.07 & 0.08 & 0.07 & 0.07 & 0.04 & 0.05 & 0.05 & - & 0.35 & 0.31 & - \\
\hline
\end{tabular}

T: Treatments; 1: C10+T20\%; 2: C10+T20\%; 3: C10+T20\%; 4: Chlorantraniliprole 20 SC; 5: Thiamethoxam 25 WG; 6: Phorate $10 \mathrm{G}$; 7: $\lambda$-cyhalothrin $5 \mathrm{EC}$; 8: Control; ${ }^{*} \mathrm{CD}$ : $\mathrm{CD}(p=0.05) ;{ }^{*}$ on $5^{\text {th }}$ and $10^{\text {th }}$ days after application no larvae of Helicoverpa armigera was recorded. Figures in parentheses are square root transformed values; DAA= Days after application

$\mathrm{q} \mathrm{ha}^{-1}$ ) during both the seasons, respectively. Next order of chemicals were chlorantraniliprole $10 \%$ +thiamethoxam $20 \%$ mixture@ 105 g a.i. ha ${ }^{-1}\left(212.33\right.$ and $\left.212.06 \mathrm{q} \mathrm{ha}^{-1}\right)$ followed by thiamethoxam $25 \mathrm{WG}\left(211.66\right.$ and $\left.209.66 \mathrm{q} \mathrm{ha}^{-1}\right)$, phorate $10 \mathrm{G}\left(209.33\right.$ and $\left.208.33 \mathrm{q} \mathrm{ha}^{-1}\right)$ and $\lambda$-cyhalothrin $5 \mathrm{EC}$ (208.66 and $207.06 \mathrm{q} \mathrm{ha}^{-1}$ ) whereas in control plots yield were 199.33 and $196.66 \mathrm{q} \mathrm{ha}^{-1}$ during both the seasons, respectively. Mean yield of tomato was also highest in chlorantraniliprole $10 \%$ +thiamethoxam $20 \%$ mixture at higher dose followed by medium and lower doses of same mixture, chloratraniliprole, thiamethoxam, phorate and $\lambda$-cyhalothrin with $214.56,231.86$, $212.86,212.19,210.66,208.83$ and $207.86 \mathrm{q} \mathrm{ha}^{-1}$, respectively whereas it was only $197.99 \mathrm{q} \mathrm{ha}^{-1}$ in control plots.

\subsection{Effects on natural enemies}

\subsubsection{Effects on encarsia $s p$}

Effects of different treatments on Encarsia sp. are presented in Table 4. It was observed that there was significant variation among the treatments regarding nymphal parasitisation of white fly by Encarsia sp. during both the seasons. During first season, lowest nymphal parasitisation of white fly by Encarsia sp was found in $\lambda$-cyhalothrin $5 \mathrm{EC}(18.69 \%)$ following phorate $10 \mathrm{G}(36.99 \%)$. chlorantraniliprole $10 \%$ thiamethoxam $20 \%$ mixture@ 150, 128 and 105 g a.i. ha ${ }^{-1}(51.03,50.79$ and $49.29 \%$, respectively), chlorantraniliprole $20 \mathrm{SC}(50.59 \%)$ and thiamethoxam $25 \mathrm{WG}(50.82 \%)$ were much safer to this natural enemy as compare to $\lambda$-cyhalothrin $5 \mathrm{EC}$ and phorate $10 \mathrm{G}$ as well as closer to control plots (52.29\%). During second season, almost similar trend of nymphal parasitisation of white fly by this parasite were found. The ascending order of parasitisation in second season were $\lambda$-cyhalothrin $5 \mathrm{EC}<$ phorate $10 \mathrm{G}<$ chlorantraniliprole 10\%+thiamethoxam 20\% mixture@150 g a.i. ha ${ }^{-1}<$ thiamethoxam $25 \mathrm{WG}<$ chlorantraniliprole $20 \mathrm{SC}<$ chlorantraniliprole 10\%+thiamethoxam 20\% mixture @ 128 g a.i. ha ${ }^{-1}<$ same mixture @105 g a.i. ha ${ }^{-1}<$ control.

\subsubsection{Effects on apantales $s p$.}

Larval parasitisation of Helicoverpa armigera by Apantales sp. varied from 7.05 to 12.06 and 7.89 to $13.23 \%$ (Table 4) during 2010-11 and 2011-12, respectively in different treatments. There were significant effects of treatments on parasitisation of Helicoverpa larvae by Apantales sp. in both the seasons. In first season, highest parasitized larvae of Helicoverpa was found in control plots (12.06\%) followed by thiamethoxam (11.89\%), chlorantraniliprole $10 \%$ +thiamethoxam $20 \%$ mixture at lower $(11.79 \%)$, intermediate (11.04\%) and higher dose (10.86\%), 
Table 4: Effect of Chlorantraniliprole10\%+ Thiamethoxam 20\% mixture against some important natural enemies associated with Tomato ecosystem

\begin{tabular}{|c|c|c|c|c|c|}
\hline \multirow[t]{2}{*}{ Treatments } & \multirow[t]{2}{*}{$\begin{array}{c}\text { Dose } \\
\left(\mathrm{g} \text { a.i. } \mathrm{ha}^{-1}\right)\end{array}$} & \multicolumn{2}{|c|}{$\begin{array}{l}\text { Per cent nymphal parasitisation of white } \\
\text { fly by Encarsia sp }\end{array}$} & \multicolumn{2}{|c|}{$\begin{array}{l}\text { Per cent larval parasitisation of } \\
\text { Helicoverpa by Apantales sp }\end{array}$} \\
\hline & & First season & Second season & First season & Second season \\
\hline $\mathrm{C} 10+\mathrm{T} 20 \%$ & 105 & $51.03(45.59)$ & $53.80(47.18)$ & $11.79(20.08)$ & $12.66(20.84)$ \\
\hline $\mathrm{C} 10+\mathrm{T} 20 \%$ & 128 & $50.79(45.45)$ & $52.69(46.54)$ & $11.04(19.41)$ & $12.33(20.56)$ \\
\hline $\mathrm{C} 10+\mathrm{T} 20 \%$ & 150 & $49.29(44.59)$ & $51.00(45.57)$ & $10.86(19.24)$ & $11.73(20.03)$ \\
\hline Chlorantraniliprole $20 \mathrm{SC}$ & 50 & $50.59(45.34)$ & $51.99(46.14)$ & $11.25(19.60)$ & $12.36(20.58)$ \\
\hline Thiamethoxam $25 \mathrm{WG}$ & 100 & $50.82(45.47)$ & $51.69(45.97)$ & $11.89(20.17)$ & $12.69(20.87)$ \\
\hline Phorate $10 \mathrm{G}$ & 1500 & $36.99(37.46)$ & $40.83(39.72)$ & $10.94(19.31)$ & $9.09(17.55)$ \\
\hline$\lambda$-cyhalothrin $5 \mathrm{EC}$ & 15 & $18.69(25.61)$ & $21.99(27.97)$ & $7.05(15.40)$ & $7.89(16.31)$ \\
\hline Control & - & $52.29(46.31)$ & $54.40(47.52)$ & $12.06(20.32)$ & $13.23(21.33)$ \\
\hline $\mathrm{CD}(p=0.05)$ & - & 0.15 & 0.09 & 0.12 & 0.13 \\
\hline
\end{tabular}

Figures in parentheses are angular transformed values

chlorantraniliprole (11.25\%), phorate (10.94\%) while lowest parasitisation was found in $\lambda$-cyhalothrin treated plots (7.05\%). During second season, lowest Apantales population was found in plots treated with $\lambda$-cyhalothrin 5 EC $(7.89 \%$ parasitisation) followed by phorate $10 \mathrm{G}(9.09 \%)$. Among the doses of chlorantraniliprole $10 \%+$ thiamethoxam $20 \%$ mixture at higher dose $\left(150 \mathrm{~g}\right.$ a.i. $\left.\mathrm{ha}^{-1}\right)$ recorded lower number of parasitized larvae (11.73\%). The same mixture @ 128 g a.i. ha $^{-1}$ was at par with chlorantraniliprole $20 \mathrm{SC}$ with 12.33 and $12.36 \%$ parasitized larvae, respectively. Chlorantraniliprole $10 \%$ +thiamethoxam $20 \%$ mixture at lower dose (105 g a.i. ha $\left.{ }^{-1}\right)$ was at par with thiamethoxam $25 \mathrm{WG}$ with 12.66 and $12.69 \%$ parasitized larvae, respectively. Effects of chlorantraniliprole on natural enemies were negligible throughout the investigation which is supported by study of Huang et al. (2011). Findings may be consistent from the study of Hussain et al. (2012) who stated that after 8-days parasitism, chlorantraniliprole resulted in maximum emergence of $T$. chilonis and it showed maximum survival (42\%) of adult at 3 hours post application.

As chlorantraniliprole $10 \%+$ thiamethoxam $20 \%$ mixture has two insecticides having different mode of action and provide effective control of both sucking and borer pests. The effect of single soil drench application against the tomato fruit borer and two sucking pests was superior over other insecticides. However, the mixture treated plots @ $150 \mathrm{~g}$ a.i. ha ${ }^{-1}$ gave maximum reductions of all the three pests compared to other chemical treatments. The second best was chlorantraniliprole 10\%+thiamethoxam 20\% mixture applied @ 128 g a.i. ha ${ }^{-1}$. The yield increase commensurated with the efficacy of various treatments being highest in the mixture applied @ $150 \mathrm{~g}$ a.i. ha $^{-1}$ closely followed by the second highest dose of the same chemical i.e. $128 \mathrm{~g}$ a.i. ha ${ }^{-1}$. Chlorantraniliprole is very effective insecticide against lepidopteran pests while thiamethoxam is an excellent chemical for controlling sucking pests. This mixture may play consequential role to suppress both lepidopteran and sucking pests through its combined effects on several insect pests. Soil drench application may give wide spectrum control protecting early season growth and provides the 'jump-start' that vegetable crops need to ensure optimal yield as well as the quality demand of the consumers.

\section{Conclusion}

Chlorantraniliprole10\%+thiamethoxam 20\% mixture@150 g a.i. ha ${ }^{-1}$ and $128 \mathrm{~g}$ a.i. ha ${ }^{-1}$ gave maximum reduction of all the three pests of tomato. There were no significant differences among the two doses. Therefore, this mixture @ $128 \mathrm{~g}$ a.i. ha $^{-1}$ may be considered for management of major insect pests of tomato.

\section{Acknowledgement}

Authors are very much thankful to the Syngenta India Ltd. for supplying formulation and providing financial support to conduct this experiment.

\section{References}

Anonymous, 2015. Horticultural statistics at a glance, National Horticulture Board, Ministry of Agriculture and Farmers Welfare, Government of India, 17.

Boselli, M., Ceredi, G., 2010. Rynaxypyr, new opportunities to control codling moth. Informatore Agrario 66, 43-47.

Chowdary, L.R., Bheemanna, M., Kumar, L.R., 2010. Bioefficacy of rynaxypyr (Coragen) $20 \mathrm{SC}$ against fruit borer Helicoverpa armigera (Hubner) in okra. International Journal of Plant Protection 3, 379-381.

David, J., Schuster and Robert, F., Morris II. 2002. Comparison 
of imidacloprid and thiamethoxam for control of the silverleaf white fly, Bemisia argentifolii and leaf miner, Liriomyza trifolii, on tomato. In: proceedings of the Florida State Horticultural Society 115, 321-329.

Hannig, G.T., Ziegler, M., Marcon, P.G., 2009. Feeding cessation effects of chlorantraniliprole, a new anthranilic diamide insecticide, in comparison with several insecticides in distinct chemical classes and mode-ofaction groups. Pest Management Science 65, 969-974.

Huang, J., Wu, S., Ye, G., 2011. Evaluation of lethal effects of chlorantraniliprole on Chilo suppressalis and its larval parasitoid, Cotesia chilonis. Agricultural Sciences in China 10, 1134-1138.

Hussain, D., Ali, A., Mushtaq-ul-Hassan, M., Ali, S., Saleem, M., Nadeem, S., 2012. Evaluation of toxicity of some new insecticides against egg parasitoid Trichogramma chilonis (Ishii) (hymenoptera:trichogrammitidae). Pakistan Journal of Zoology 44, 1123-1127.

Lange, W.H., Bronson, L., 1981. Insect pests of tomatoes. Annual Review of Entomology 26, 345-71.

Lee, Y.S., Lee, S.Y., Park, E.C., Kim, J.H., Kim, G.H., 2002. Comparative toxicities of pyriproxyfen and thiamethoxam against the sweet potato whitefly, Bemisia tabaci (Homoptera:Aleyrodidae). Journal of Asia-Pacific
Entomology 5, 117-122.

Murphy, S., La Salle, J., 1999. Balancing biological control strategies in the IPM of new World invasive Liriomyza leaf miners in field vegetable crops. Biocontrol News and Information. 20, 91N-104N.

Oliveira, M.R.V., Henneberry, T.S., Anderson, P., 2001. History, current status and collaborative research projects for Bemisia tabaci. Crop Protection 20(9), 709-723.

Sastry, K.S.M., Singh, S.J., 1979. Control of the spread tomato leaf curl virus by controlling the white fly population. Indian Journal of Horticulture 31, 178-182.

Tewari, G.C., Krishnamoorthy, P.N., 1984. Yield loss in tomato caused by fruit borer. Indian Journal of Agricultural Sciences 54, 341-343.

Vyas, H.N., Saxena, H.P., 1981. Comparative efficacy of phorate, disulfoton and carbofuran against leaf miner, Phytomyza horticola Goureau (=atricornis) infesting peas (Pisum sativum L.). Indian Journal of Plant Protection 9, 56-60.

Yang, L.W., Chen, J.Z., Dai, Y.T., Ding, L.W., Yang, J.W., 2010. Control effect of three new amide pesticides on rice leaf folder, Cnaphalocrocis medinalis, at the booting stage of rice. Chinese Bulletin of Entomology 47, 393-395. 